\title{
How many causal pathways must symptoms form before we call them a borderline? A hierarchical network model of borderline personality disorder
}

\author{
Zsolt Unoka, MD, $\mathrm{PhD}^{1}$, Mara J. Richman, $\mathrm{MSc}^{2 *}$, Dániel Czégel, MSc ${ }^{3,4}$
}

1. Department of Psychiatry and Psychotherapy, Semmelweis University, Budapest, Hungary

2. Department of Clinical Psychology and Addiction, Eötvös Loránd University, Budapest, Hungary

3. MTA Center for Ecological Research, Evolutionary Systems Research Group, Tihany, Hungary

4. Department of Plant Systematics, Ecology and Theoretical Biology, Eötvös Loránd University, Budapest, Hungary

\footnotetext{
*Correspondence: Mara J. Richman, Eötvös Loránd University, Department of Addiction and Clinical Psychology, Izabella utca 46, Budapest, Hungary, 1065; Email: mara.richman@ppk.elte.hu; Phone Number: 36-30-290-8434
} 


\begin{abstract}
Borderline personality disorder (BPD) is characterized by impulsivity, emotion dysregulation, disturbed relationships, and identity disturbances. Despite the known variable co-occurrence of BPD symptoms, the possible causal relationships are not well understood. We addressed this by creating a hierarchical network model of BPD, which identifies the most likely acyclic causal pathways that are driving BPD development. Cross-sectional data was obtained from the Structured Clinical Interview-II (SCID-II), and possible causal relationships between symptoms were identified from conditional independence relations. The symptoms' hierarchy values, assessing their role in causal pathways, was determined by a random walk-based algorithm. By analyzing the directed network of BPD symptoms, it was found that symptoms in initial stages of causal pathways were abandonment, physical fights, impulsivity, suicidal threats, identity disturbances, and affective instability. Based on the assessed role symptoms play in causal pathways of BPD development, specific symptoms can be targeted during early diagnosis and clinical assessment.
\end{abstract}

Keywords: borderline personality disorder, symptom network, diagnosis, causal model 


\section{Introduction}

Borderline personality disorder (BPD) is defined in two ways in the Diagnostic Statistical Manual (DSM-5; American Psychiatric Association (APA, 2013): categorical and dimensional. In the categorical model, BPD is described as a qualitatively distinct clinical syndrome or a group of symptoms that appear together and are not better explained by another mental disorder (APA, 2013). In the dimensional model, BPD is referred to as a specific constellation of personality disorder traits and areas of personality dysfunction with different levels of severity (APA, 2013). Both models are consistent with the traditional concept of disease in medicine; there is an underlying BPD disorder, a yet unknown disease that explains a) the covariation of symptoms in the categorical model, or b) the co-variation of latent trait dimensions, which in turn explain the variation of items designed to measure those traits.

Both the disease model and the latent variable model assume a vertical causation structure, where the symptoms are caused by hidden factors. These hidden factors can be found by assuming a given mathematical model of the data and using the corresponding dimension reduction technique (e.g., factor analysis). However, these models cannot account for the causal relationship between the symptoms themselves. Many studies have tested the factor structure of the DSM-IV (APA, 2013) criteria for BPD by using exploratory and hypothesis driven confirmatory factor analyses of cross-sectional data of structured personality disorder interviews (Sanislo et al., 2002). For example, Sanislo et al., 2012 used confirmatory factor analysis and hypothesized that affective instability, inappropriate anger, and fear of abandonment are causally dependent on a latent factor called "affective dysregulation" or at least this factor represents the common elements shared by these three symptoms. On the other hand, the same authors proposed another theoretical possibility- that there may be horizontal causal relations between 
latent constructs or symptoms and "for instance, affective dysregulation may moderate behavioral impulses" (Sanislo et al., 2002)

Another model of personality disorders can be considered called the network model of BPD. The model supposes that structural co-variation of BPD symptoms may be the result of direct interactions between symptoms as measured through personality disorder interviews. This model offers an alternative way to analyze personality data, which is not through the a priori imposition of a latent variable structure but through the construction of a network that represents the most important relations between symptoms. For example, it can be supposed that people who are very sensitive to any minor form of rejection will make frantic efforts to avoid real or imagined abandonment, and in turn, they will react to perceived abandonment with sudden mood changes. As such, personality may resemble an ecosystem in which each characteristic could stimulate or inhibit the other (Costantini et al., 2015). In our network model, symptoms constitute BPD rather than being caused by it. Furthermore, in our network model we do not just measure the symmetric relationships between symptoms but even we consider the causal pathways between symptoms. It can be speculated that BPD symptoms may be strongly correlated because they causally influence one another.

Networks consist of two building blocks: nodes and edges (Borsboom and Cramer, 2013). In case of cross-sectional data of structured personality disorder interviews, the nodes are the symptoms usually visualized as circles and edges or links are lines that connect these nodes representing a symmetric relationship, e.g., correlation between symptoms. In addition, if it is somehow possible to infer causal relationships between the symptoms, they can be represented as directed edges instead of undirected ones, e.g. depicting them by arrows instead of simple lines. In this case, the network itself is also called directed. Kalisch et al. (2016) implemented an 
algorithm (available in R's pcalg package) that can infer information about the causal structure of cross-sectional, observational data by identifying conditional independence relationships between the observed variables. Although conditional independence models have been mostly used to test hypotheses on causal structures, Spirtes et al. (2000) introduced an algorithm by which these models can be used in an explorative way, resulting in candidate causal structures that might be responsible for the patterns among the observed variables. Danks et al. (2010) suggested using this exploratory data analysis technique in psychopathology, where highresolution longitudinal data are rarely available. A detailed explanation along with simple examples on the usage of conditional independence models in psychopathology can be found in Borsboom and Cramer (2013). According to our best knowledge, there is no study that built a directed network from cross-sectional BPD symptom data.

In order to clarify to what extent a given symptom is in the beginning of any causal pathway in the directed network, the study applied a relatively new mathematical method called "random walk hierarchy measure" developed by Czégel and Palla (2015). This method is able to quantify how hierarchical a (possibly weighted and directed) network structure is by using random walkers to explore the network. Moreover, it does so by first identifying those elements that are mostly sources of anything that can spread in the network (e.g., information), and those which are mostly the receivers. This provides us a natural measure of how much a given symptom is in the beginning of any causal chain. Overall, these two methods namely, the construction of a directed network from cross-sectional data and a hierarchy measure, allows us to analyze and visualize the BPD symptoms' overall causal structure and to identify the most probable pathways of disease development. 
In the current study, we constructed a causal network model of BPD by using crosssectional observational data. We used a statistical model based on conditional independence relations between the BPD symptoms to create the directed network of them. Second, we measured the extent to which a given symptom is causing other symptoms rather than being caused by other symptoms. To accomplish these goals, we analyzed the DSM-IV criteria for BPD that we had obtained from semi structured clinical interviews in the SCID-II [9]. This diagnostic information was obtained from assessments of psychotherapeutic treatment seeking personality-disordered subjects who were recruited as part of a cross-sectional study of socialnetwork of patients suffering from personality disorders.

Estimating causal relations based on cross-sectional data is a highly non-trivial task. Therefore, it is instructive to clarify the main underlying assumptions and the possible limitations of the model we use in this paper. First, it assumes that the symptoms form a closed system of conditional dependence relations (possibly originating from causal dependencies), without reference to external variables that might in reality very well trigger the expression of symptoms in the first place. Second, the model searches for the best acyclic structure that accounts for the data, which renders closed directed loops a priori out of scope. Third, the model is fed with aggregated data, and accordingly, it is not suitable for explaining individual pathways of disorder development. Fourth, it assumes that there are no hidden variables that might (partly) explain the structure of the data. Consequently, our analysis does not compare causal symptom network models with hidden factor models, and therefore, does not contribute to the general debate of whether which of these two model structures explain symptom data better. 


\section{Subjects}

\section{Methods}

Subjects included in the study were 341 subjects diagnosed with personality disorders according to the DSM-IV-R (APA, 2000). Patients were inpatients in a four-week cognitive psychotherapy program in the Department of Psychiatry and Psychotherapy at Semmelweis University in Budapest, Hungary. Participation in the study was voluntary, and compensation was not provided. Informed consent was obtained from all subjects. All procedures contributing to this work comply with the ethical standards of the relevant national and institutional committees on human experimentation and with the Helsinki Declaration of 1975, as revised in 2008. The Local Ethical Committee of the Semmelweis University approved the study protocol.

\section{Clinical assessment}

To confirm personality disorder diagnostic status, the Hungarian version of the Structured Clinical Interview for DSM-IV Axis II Personality Disorders (SCID-II; Szádóczky et al., 2004) was administered. Assessment was conducted in Hungarian and carried out by a trained, experienced psychiatrist and a masters or PhD level clinician.

Exclusion Criteria. Subjects were excluded if a) they met criteria for a current or past diagnosis of schizophrenia, schizoaffective disorder, a psychotic disorder, an organic mental syndrome, a central nervous system neurological disease, current hypomanic/manic episode, and/ or substance withdrawal syndrome and/or b) they did not have the mental competency and ability to give informed consent and/or c) they had been abusing illicit substances and alcohol for at least two weeks at the time of the assessment.

\section{Data Analysis}


SCID II data. We used the questions 90-104 from the SCID-II for personality disorders. In the SCID-II interview, there are 15 questions for the nine BPD symptoms. Identity disturbances are operationalized with four questions, suicidal and self-harming behavior with two questions, and inadequate anger with three questions. The patient's answer to any of the symptom-related questions was rated 3 (threshold or true) by the clinician if the patient had given several examples of the symptom and the symptom leads dysfunctionality in the patient's life. An item was rated 2 in the case that the there was a sub-threshold presence of the trait. An item was rated 1 if the symptom was absent or the patient answers and examples did not fit to the definition of the symptom. We used binary data for the analysis and therefore divided ratings into two categories: the threshold or true symptoms (0) and sub-threshold or absence of a symptom (1). The authors met to select a general symptom term in BPD that could be assigned to each question. After a general symptom term was chosen, a shortened word was chosen to fit in a visual rendering of the directed network of the symptoms.

Construction of the correlation network of BPD symptoms: In order to illustrate the different nature of information that can be extracted from the same data by forming a causal network of symptoms compared to computing a simple symmetric association measure, we constructed the correlation network of the BPD symptoms. The correlation network is a weighted, undirected network where the nodes represent the BPD symptoms, while the thickness and the grayscale of the edges indicate the Pearson correlation value between the corresponding symptoms.

Construction of the directed network of symptoms. The construction of the directed network representing causal relationships consists of two main steps. First, the so-called skeleton of the network is determined which is an undirected network of the same symptoms where symptoms $i$ and $j$ are connected if and only if they are conditionally dependent. Formally, $i$ and $j$ are 
conditionally dependent if the following null hypothesis can be rejected at significance level $p=0.05$ for every possible subset $S$ of the remaining symptoms: $i$ and $j$ are conditionally independent, given $S$. Then, as a second step, the algorithm assigns directionality to each of the links in the skeleton if the directionality can be unambiguously determined, based on the following idea: for every such $i, j, k$ symptom triplets in the network where $i$ is connected to $j$, and $j$ is connected to $k$, but $i$ is not connected to $k$ (i.e., $i-j-k$ ), the links are directed towards $j$ (i.e., $i->j<-k$, called a v-structure), if and only if $j$ was not in the set $S$ which caused the lack of connection between $i$ and $k$ in the skeleton. For the remaining undirected edges, there are alternative strategies by which one can infer their directionality, such as the fact that the resulting network should not contain any directed cycle nor should new v-structures be introduced. However, edges whose directionality cannot be unambiguously determined might still remain; these edges are depicted without any arrow. For the details of the algorithm (binCItest), see the documentation of the pcalg package (Kalish et al., 2016), and the references therein.

Determination of the symptoms' position in the hierarchy. In order to assign a value $h$ to each symptom which determines to what extent the given symptom is in the beginning of any causal chain, or, in terms of information spreading processes, how much the given symptom is a source of any piece of information, we used a method based on random walks on the network (Czégel and Palla, 2015). The value $h$ is determined in the following way: random walkers traversing through the network for infinite number of time steps, following the directed edges backwards. Every time-step consists of three actions:

a. $f$ random walkers are distributed among the symptoms uniformly (that is, the number of random walkers at every symptom is increased by $f / N$, where $N$ is the number of symptoms; here we allow for having non-integer number of walkers at a symptom. 
By this one can avoid being stuck at one of the root symptoms (having only outgoing edges), and thus we will be able to distinguish between networks not only based on the number of root symptoms, but based on the general structure of the network.

b. Every walker, being at node $j$, crawls through one of the incoming edges $i \rightarrow j$ backwards, with probability $P(j \rightarrow i)=\frac{1}{k_{j}^{\text {in }} k_{i}^{\text {out }}}$ and stays at that same symptom with the remaining probability.

c. The number of walkers in the network is normalized to 1 , that is, the number of walkers at every symptom is divided by $1+f$.

Then, the value $h(i)$ of symptom $i$ equals to the relative frequency symptom $i$ is visited by any walker during the infinite process, and can be mathematically determined as the stationary probability distribution of the Markov chain having the above defined transition probabilities. For mathematical details and other properties of the algorithm, see (Czégel and Palla, 2015). A free parameter of the model is the number of walkers $f$ injected to the system at every time step. In the following, $f$ is set such that the decay time constant of the random walkers is unity, i.e., any unit amount of walkers decays to $e^{-1}$ during one time step as the effect of normalization. Hence, $\mathrm{f}$ can be determined from $\frac{1}{1+f}=e^{-1}$, being $f=e-1$. 


\section{Results}

\section{Patient SCID descriptive statistics}

The mean number of the questions that were rated 3 was $5.31(\mathrm{SD}=4.18)$. As for $\mathrm{BPD}$ diagnosis, 113 patients met DSM-IV (APA, 2000) criteria (at least five symptoms of the nine), whereas 167 had at least one symptom for the disorder, but they did not meet full criteria. The remaining 87 participants did not have any BPD symptoms. The symptoms for the directed network as paired with the number of participants per symptom can be seen in table 1 .

In the figure depicting the network, the authors decided use the following abbreviations to designate the 15 SCID questions matching the symptoms from the DSM-IV: a. Frantic effort to avoid real or imagined abandonment (aband) b. unstable relationships (unstRel), c. Identity disturbance: sudden change of the sense of who the patient is and what is his/her goal (IDSud), d. Identity disturbance: frequent change of the sense of who the patient is and what is his/her goal (IDFreq), e. Identity disturbance: different views of self with different people in different situations (IDdiff), f. Identity disturbances: sudden changes in core identity topics such as long term goals, career plans, religion (IDchan), g. impulsivity (impuls), h. suicidal threats (suicThr), i. self-mutilation (selfMut), j. affective instability due to a marked reactivity of mood, sudden mood change (UnstAff), k. emptiness (empty), 1. Anger: difficulty in controlling anger (angerCont), m. Anger: physical fights as hitting people or throwing things (physFight), n. Anger: minor things make them very angry (minAng), p. Severe dissociative symptoms and transient stress related paranoid ideations (DissPar). It is important to note that in the SCID-II interview three BPD symptoms (identity disturbance, suicidal and self-harming behavior, and inadequate anger) were operationalized with more than one question. 


\section{Correlation matrix and network:}

Figure 1 shows the undirected correlation network between BPD SCID-II items and the bar legend of the strength of the correlations.

\section{Causal Networks:}

Figure 2 displays the directed network between the symptoms. By combining recent methods of statistical modeling and network analysis, the authors identified sets of symptoms that are causing other symptoms, being caused by other symptoms, and/or transmitting between different symptoms. Abandonment, physical fights, impulsivity, suicide threats and self-harm, one aspect of identity disturbance (sudden change of goals) caused other symptoms. Whereas paranoid and dissociative symptoms and one aspect of identity disturbance (sudden change of the sense of who the patient are) symptoms are being caused by other symptoms but they themselves do not cause other symptoms. Transmitting symptoms, that is symptoms that are causing other symptoms and being caused by other symptoms, are unstable relationships, affective instability, emptiness, inability to control anger, and minor things provoking anger. Symptoms that are connected to edges without any arrow the directionality of causal relation were not unambiguously determined: edges between aspects of identity disturbances (IDwho: sense of identity often change; idDiff: different with different people; IDgoal: sudden change in sense of identity); an edge between suicidal attempts/threat (suicThr), and self-mutilating behavior (selfMut).

\section{Hierarchy values}

Hierarchy values can be seen in table 2 . It can be noted that there is a wide distribution of hierarchy values. Frantic effort to avoid real or imagined abandonment $(h=.13)$, anger-physical fights as hitting people or throwing things $(h=.13)$, impulsivity $(h=.12)$, suicide threats $(h=.1)$, identity disturbances: sudden change of the sense of who the patient are $(h=.09)$, affective 
instability due to a marked reactivity of mood $(h=.08)$ were shown to have the highest hierarchy value- meaning these symptoms are with the highest extent at the beginning of any causal chain. Severe dissociative symptoms and transient stress related paranoid ideations $(h=.01)$, emptiness $(h=.01)$, and inappropriate anger (minor things make them very angry $(h=0.02)$ were shown to have the lowest hierarchy value. That is, these symptoms are with the lowest extent at the beginning of any causal chain. These results allow us to see the most related first starts of causal relationships between the network of the BPD symptomatology. 


\section{Discussion}

To our knowledge, this is the first study that examined an empirical directed network structure of BPD symptoms as assessed with the SCID-II interview. The first aim of our study was to understand the causal relationships between symptoms of BPD by inferring causal information from observational data. By identifying conditional independence relationships, inferred information about the causal structure of BPD symptoms from cross-sectional, observational data of SCID-II ratings can be found. With this methodology, it is assuming that the overall organization of the symptoms of BPD may arise from the causal relations of the symptoms themselves (Boorsboom and Cramer, 2013) as opposed to the traditional models assuming one, three, or four hidden BPD factors (Michonski, 2015). The second aim of our study was to further understand the overall causal structure of BPD symptoms by defining each symptom's hierarchy value in the causal chains by using a method based on random walks in the network (Czégel and Palla, 2015).

First, the authors identified the network structure of BPD symptoms. In analyzing the directed network of BPD symptoms, the authors found that the symptoms are forming causal chains. Further we identified which symptoms are causing other symptoms, being caused by other symptoms, and/or transmitting between different symptoms. Abandonment, anger-related physical fights, impulsivity, suicidal/self-mutilating behaviors, and identity disturbances have only outgoing arrows, which suggests that these symptoms might come earlier to developing BPD by triggering the development of other symptoms. Unstable relationships, affective instability, emptiness, some aspects of anger (i.e., difficulty in controlling anger; minor things provoke anger) are transmitting symptoms. Stress-related paranoid ideation/dissociation and identity disturbances (IdChange: sudden changes in core identity topics: long term goals, career 
plans, religion) are triggered by other symptoms, but they themselves do not cause other symptoms. It was clear from our data that symptoms that were operationalized by more than one item in the SCID-II such as inappropriate anger, identity disturbances, and suicidal/self-harming behavior were highly interconnected in the network and in case of the latter two the directionality of causal relation were even not unambiguously determined; on the other hand, different aspects of the symptoms have different roles in the causal chains of the network.

Second, it was examined to what extent each BPD symptom plays a causal role in the network. The symptoms with the highest hierarchy value, signaling that these symptoms are mostly causing others than being caused by others, were the following: frantic effort to avoid real or imagined abandonment, anger-physical fights (such as hitting people or throwing things), impulsivity, suicide threats, identity disturbances (dramatic change in sense of who the patient is), and affective instability due to a marked reactivity of mood. We also identified symptoms that are more caused by other symptoms than are causing them; those were severe dissociative symptoms and transient stress related paranoid ideations, emptiness, anger (minor things make cause extreme anger), and sudden changes in core identity (topics such as long term goals, career plans, religion).

The interpersonal hypersensitivity causal chain starts with the symptom of frantic efforts to avoid abandonment, and it triggers unstable relationships and stress-related paranoid ideation/dissociation. Unstable relationships also have a relatively high hierarchy value and trigger two symptoms: affective instability and identity (sudden changes in core identity topics: long term goals, career plans, religion). In our network model of BPD, the causal chains that start from abandonment can reach the most symptoms. This finding is in line with previous research, which showed that attachment system disturbances are core features of BPD. On a neuropeptide 
level, opioids, oxytocin, and vasopressin systems may be affected (Stanley and Siever, 2010); on a developmental level early separation in prospective (Crawford et al., 2009) and retrospective (Bandelow et al., 2005; Links et al., 1988; Reich and Zanarini, 2001; Soloff and Millward, 1983) studies, chaotic, invalidating, critical family environment (Fruzzetti et al., 2005), biparental neglect and emotional denial (Zanarini et al., 1997) were associated with BPD; on the trait level insecure attachment (Agrawal et al., 2004; Levy, 2005), rejection sensitivity (Ayduk, et al, 2008; Staebler, et al., 2011), distrust (King-Casas et al., 2008; Unoka et al., 2007; Miano et al., 2013) characterize BPD; and finally in factor analytic studies, disturbed relationships factor were continually found (Michonski, 2015). Identity disturbances are first causes in our network model with a high hierarchy value and was caused by unstable relationships. Identity disturbances distinguishes patients with BPD from other patients (Wilkinson-Ryan and Westen, 2000), and further it is observable from adolescence to be distinctively associated with BPD (Richman, 2014; Westen et al., 2011).

Affective instability had a relatively high hierarchy value and was caused by suicidal/self-mutilating behaviors. In addition, it caused emptiness which triggers stress-related paranoid ideation/dissociation. Affective instability in BPD patients often occurs in an interpersonal context and is often precipitated by real or imagined events in relationships. Together, it can rapidly lead to impulsive, aggressive, self-destructive behavior, or dissociative states (Yeomans and Levy, 2002; Levy et al., 2007; Berenson et al., 2011). Linehan and Heard (1999) proposed the reversed direction of causal association, however, previous findings did not confirm the hypotheses that affective instability leads to self-destructive behaviors (Links et al., 2007). Links et al. (2007) found that negative mood intensity was that significantly related to patients' self-reported suicidal ideation and to suicidal behavior. Negative mood intensity or 
dysphoric affect (Zanarini et al, 1998) may be an important first cause (which induces suicidal/self-harming thoughts and intentions) that is missing from DSM-V categorical definition of BPD (APA, 2000).

Self-mutilations as well as suicidal threat gestures and behavior are first causes in our causal chain model. Our results point to the tendency to be self-destructive may be an independent phenomenon, a first link in one causal chain in BPD. In a study by Zanarini et al., (2006), it was found that one third of BPD patients began self-harming as early as 12 years old or younger. Among adults the prevalence and the frequency of these self-destructive behaviors declines significantly over time, however, they remain significantly more common among BPD patients compared to other personality disorders (Zanarini et al., 2006). At least three-quarters of BPD patients attempt suicide and approximately 10\% eventually complete suicide (Black et al., 2003). Cross-sectional rates of self-mutilation have ranged from $17-80 \%$ (median= 53\%) while suicide attempts have ranged from 46-92\% (median=76\%) and suicidal and self-harming behavior frequently overlap [36]. These results also imply that a significant amount of BPD patients does not have self-destructive behavior. Based on our results, we can say that selfmutilations and suicidal threats, gestures, and behavior may be an independent causal factor in $\mathrm{BPD}$, or may relate other symptoms, for example depressive symptoms, which are not part of the DSM-IV BPD criteria.

The other two important first causes in the directed network with high hierarchy values are impulsivity and anger- related physical fight—both trigger inappropriate anger (minor things provoke anger), which triggers stress-related paranoid ideation/dissociation. Together, this is also caused by inability to control anger which was triggered by anger-related physical fights. Angerrelated physical fights and impulsivity were also at the beginnings of causal chains. These results 
are partly in accordance with Siever and Davis's (1991) model of BPD that proposes two temperament factors, impulsive aggression and affective lability, which predispose people to develop BPD symptomatology. In the prospective study of Crawford et al. (2009), angry tantrums at age nine predicted BPD symptoms in adolescence and adulthood. In other previous studies, impulsiveness and aggressiveness were very characteristic of BPD (Virkkunen, 1976; Pattison and Kahan, 1983; New et al., 1999; Henry et al., 2001). Labile anger alone was sufficient to predict diagnosis of BPD versus another personality disorder with a $72 \%$ accuracy (Koenigsberg et al., 2002).

Stress related paranoid ideations and dissociations was a symptom that had the lowest hierarchy value and was being caused by other symptoms, however, it alone did not cause other symptoms. These results fit into the theoretical concept of dissociation that serves as a defensive flight from either aloneness (Gunderson, 1984) or feelings of emptiness (Linehan, 1993). Other empirical results also found a strong correlation between induced aversive tension and dissociative symptoms in BPD (Stiglmayr et al., 2001).

Further, our results showed that different causal chains connect BPD symptoms and that the causal network model of BPD is a reasonable model. Our model could give another explanation to the heterogeneity of BPD. BPD may be a heterogeneous syndrome because the first unit of a causal chain could start from different symptoms such as externalizing ones referring to impulsivity, inappropriate anger or internalizing ones as affective instability due to mood reactivity or relational ones as abandonment and unstable relationships, self-destructive behaviors and identity disturbances.

If symptoms are causally related, then it is important to define the first unit of a causal chain as a target of a therapeutic intervention. If we can alleviate a specific symptom, it may lead 
to the decrease in the severity of other symptoms. Different therapeutic interventions target different symptoms of BPD. For a pharmacological example, pretreatment trait impulsivity symptoms and state aggression symptoms predict a favorable response to divalproex relative to placebo for impulsive aggression, however, baseline affective instability did not influence differential treatment response in patients with borderline personality disorder (Hollander et al., 2005). In differing psychotherapeutic treatments of BPD, there are dissimilar targets of interventions. For example, disturbed relationships are the focus of mentalization based therapy (MBT), whereas identity disturbances are the focus of transference focused therapy (TFP), and for self-destructive behavior and affect-regulation dialectical behavioral therapy (DBT) is a major psychotherapeutic treatment; although these psychotherapeutic methods are not so narrowly targeted and their influences go far beyond their focuses.

\section{Limitations}

One limitation of the present study is that we used SCID-II data that are based on the DSM-IV symptoms. In the DSM-IV, BPD is defined by symptoms that are either stable personality traits (impulsivity) or dysfunctional behaviors that are attempts at coping with pathological traits (dissociations) (Skodol et al., 2005). For example, in the case of the abandonment symptom, it would have been more reasonable to apply our symptom network model to a database where the trait of separation insecurity and the way a person copes would have been two independent items. Furthermore, here we emphasize the methodological assumptions that limit the applicability of the inferences drawn based on our results. The most important such assumption is that model we applied does not take into account either external variables nor hidden variables. Consequently, our analysis does not provide information 
regarding external variables that might be responsible for triggering the activity of the symptoms, nor does it compare symptom network models to hidden variable models. Moreover, the conditional dependence structures that form the basis of the directed symptom network are acyclic, implying that the model is incapable of representing circular causal activation patterns of the symptoms. This method, applied to our dataset, is also unsuitable to model individual differences of disorder pathway development. 


\section{References}

Agrawal HR, Gunderson JG, Holmes BM, Lyons-Ruth K (2004) Attachment studies with borderline patients: A review. Harvard Review of Psychiatry. 12, 94-104

American Psychiatric Association. (2000). Diagnostic and statistical manual of mental disorders (4th ed.). Washington, DC: Author.

American Psychiatric Association. (2013). Diagnostic and statistical manual of mental disorders (5th ed.). Washington, DC: Author.

Ayduk, Ö, Zayas, V, Downey, G, Cole, AB, Shoda, Y, Mischel, W (2008). Rejection sensitivity and executive control: Joint predictors of borderline personality features. Journal of Research in Personality, 42, 151-168.

Bandelow, B, Krause, J, Wedekind, D, Broocks, A, Hajak, G, Rüther, E. (2005). Early traumatic life events, parental attitudes, family history, and birth risk factors in patients with borderline personality disorder and healthy controls. Psychiatry Research, 134 169-179.

Berenson, KR, Downey, G, Rafaeli, E, Coifman, KG, Paquin, NL (2011) The rejection-rage contingency in borderline personality disorder. Journal of Abnormal Psychology, 120, 681.

Borsboom, D, Cramer, AO (2013). Network analysis: an integrative approach to the structure of psychopathology. Annual review of clinical psychology, 9, 91-121.

Clarkin, J F, Hull, JW, Hurt, SW (1993). Factor structure of borderline personality disorder criteria. Journal of Personality Disorders, 7,137-143. 
Costantini, G, Epskamp, S, Borsboom, D, Perugini, M, Mõttus, R, Waldorp, LJ, Cramer, AO (2015). State of the aRt personality research: A tutorial on network analysis of personality data in R. Journal of Research in Personality, 54, 13-29.

Crawford, TN, Cohen, PR, Chen, H, Anglin, DM, Ehrensaft, M (2009). Early maternal separation and the trajectory of borderline personality disorder symptoms. Development and psychopathology, 21, 1013-1030.

Czégel, D, Palla, G (2015). Random walk hierarchy measure: What is more hierarchical, a chain, a tree or a star? Scientific reports, 5

Danks D, Fancsali S, Glymour C, Scheines R (2010). Comorbid science? Behav. Brain Sci. 33:153-54

First, MB (1997). User's guide for the structured clinical interview for DSM-IV axis II personality disorders: SCID-II. American Psychiatric Pub.

Fruzzetti, AE, Shenk, C, Hoffman, PD (2005). Family interaction and the development of borderline personality disorder: A transactional model. Development and psychopathology, 17(04), 1007-1030.

Henry, C, Mitropoulou, V, New, AS, Koenigsberg, HW, Silverman, J, Siever, LJ (2001). Affective instability and impulsivity in borderline personality and bipolar II disorders: similarities and differences. Journal of psychiatric research, 35, 307-312.

Koenigsberg, HW, Harvey, PD, Mitropoulou, V, Schmeidler, J, New, AS, Goodman, M, ... Siever, LJ (2002). Characterizing affective instability in borderline personality disorder. American Journal of Psychiatry, 159, 784-788.

Levy, KN (2005). The implications of attachment theory and research for understanding borderline personality disorder. Development and Psychopathology, 17, 959. 
Linehan, M (1993). Cognitive-behavioral treatment of borderline personality disorder. Guilford press.

Linehan MM, Heard HL. (1999) Borderline personality disorder: Costs, course, and treatment outcomes. In: Miller N, Magruder K, editors. The Cost Effectiveness of Psychotherapy: A Guide for Practitioners. New York: Oxford University Press

Links, PS, Eynan, R, Heisel, MJ, Barr, A (2007). Affective instability and suicidal ideation and behavior in patients with borderline personality disorder. Journal of personality disorders, 21, 72.

Links, PS., Steiner, M, Offord, DR, Eppel, A (1988). Characteristics of borderline personality disorder: a Canadian study. The Canadian Journal of Psychiatry, 33, 336340.

Kalisch, M, Maechler, M, Colombo, D, Maathuis HM, Buehlmann, P (2012). Causal Inference Using Graphical Models with the R Package pcalg. Journal of Statistical Software, 47

Miano, A, Fertuck, EA, Arntz, A, Stanley, B (2013). Rejection sensitivity is a mediator between borderline personality disorder features and facial trust appraisal. Journal of personality disorders, $27,442$.

Michonski, JD (2014). The underlying factor structure of DSM criteria in youth BPD. In C. Sharp JL Tackett (Eds.), Handbook of borderline personality disorder in children and adolescents (pp. 35-48). New York: Springer

New, AS, Sevin, EM, Mitropoulou, V, Reynolds, D, Novotny, SL, Callahan, A, ... Siever, LJ (1999). Serum cholesterol and impulsivity in personality disorders. Psychiatry research, 85, 145-150. 
Pattison, EM, Kahan, J (1983). The deliberate self-harm syndrome. American Journal of Psychiatry, 140, 867-872.

Richman, MJ (2014). Subtypes of Identity Disturbance and Their Association with the Course of Borderline Personality Disorder Over 16 Years of Prospective Follow-up. Kalamazoo College Psychology Senior Individualized Projects Collection, http://hdl.handle.net/10920/30047

Sanislow, CA, Grilo, CM, Morey, LC, Bender, DS, Skodol, AE, Gunderson, JG., ... McGlashan, TH (2002). Confirmatory factor analysis of DSM-IV criteria for borderline personality disorder: findings from the collaborative longitudinal personality disorders study. American Journal of Psychiatry, 159, 284-290.

Siever, LJ, Davis, KL (1991). A psychobiological perspective on the personality disorders. American journal of Psychiatry, 148, 1647-1658.

Skodol, AE, Gunderson, JG, Pfohl, B, Widiger, TA, Livesley, WJ, Siever, LJ (2002). The borderline diagnosis I: psychopathology, comorbidity, and personality structure. Biological psychiatry, 51, 936-950.

Skodol, AE, Gunderson, JG, Shea, MT, McGlashan, TH, Morey, LC, Sanislow, CA., ... Pagano, ME (2005). The collaborative longitudinal personality disorders study (CLPS): Overview and implications. Journal of personality disorders, 19, 487.

Soloff, PH, Millward, JW (1983). Psychiatric disorders in the families of borderline patients. Archives of General Psychiatry, 40(1), 37-44.

Spirtes P, Glymour CN, Scheines R (2000) Causation, Prediction, and Search. Cambridge, MA: MIT Press 
Staebler, K, Helbing, E, Rosenbach, C, Renneberg, B (2011). Rejection sensitivity and borderline personality disorder. Clinical psychology psychotherapy, 18, 275-283.

Stanley, B, Siever, LJ (2009). The interpersonal dimension of borderline personality disorder: toward a neuropeptide model. American Journal of Psychiatry, 167, 24-39.

Stiglmayr, CE, Shapiro, DA, Stieglitz, RD, Limberger, MF, Bohus, M (2001). Experience of aversive tension and dissociation in female patients with borderline personality disorder - a controlled study. Journal of psychiatric research, 3, 111-118.

Szádóczky, E, Unoka, Z, Rózsa, S (2004). User's Guide for the Structured Clinical Interview for DSM-IV Axis II Personality Disorders (SCID-II), Hungarian Version. OS Hungary Kft.

Unoka, Z, Seres, I, Áspán, N, Bódi, N, Kéri, S (2009). Trust game reveals restricted interpersonal transactions in patients with borderline personality disorder. Journal of Personality Disorders, 23(4), 399.

Virkkunen, MA (1976). Attitude to psychiatric treatment before suicide in schizophrenia and paranoid psychoses. The British Journal of Psychiatry, 128(1), 47-49.

Westen, D, Betan, E, DeFife, JA (2011). Identity disturbance in adolescence: Associations with borderline personality disorder. Development and psychopathology, 23, 305-313.

Wilkinson-Ryan, T, Westen, D (2000). Identity disturbance in borderline personality disorder: An empirical investigation. American Journal of Psychiatry, 157(4), 528541.

Yeomans FE, Levy KN (2002) An object relations perspective on borderline personality disorder. Acta Neuropsychiatr, 14, 76-80. 
Zanarini, MC, Frankenburg, FR. (1997). Pathways to the development of borderline personality disorder. Journal of personality disorders, 11, 93-104.

Zanarini, MC, Frankenburg, FR. DeLuca, C.J., Hennen, J, Khera, GS, Gunderson, JG (1998). The pain of being borderline: dysphoric states specific to borderline personality disorder. Harvard Review of Psychiatry, 6, 201-207.

Zanarini, MC, Frankenburg, FR, Hennen, J, Reich, DB., Silk, KR. (2005). The McLean Study of Adult Development (MSAD): overview and implications of the first six years of prospective follow-up. Journal of personality disorders, 19, 505-523.

Zanarini, MC, Frankenburg, FR., Ridolfi, ME, Jager-Hyman, S, Hennen, J, Gunderson, JG (2006). Reported childhood onset of self-mutilation among borderline patients. Journal of personality disorders, 20, 9-15. 


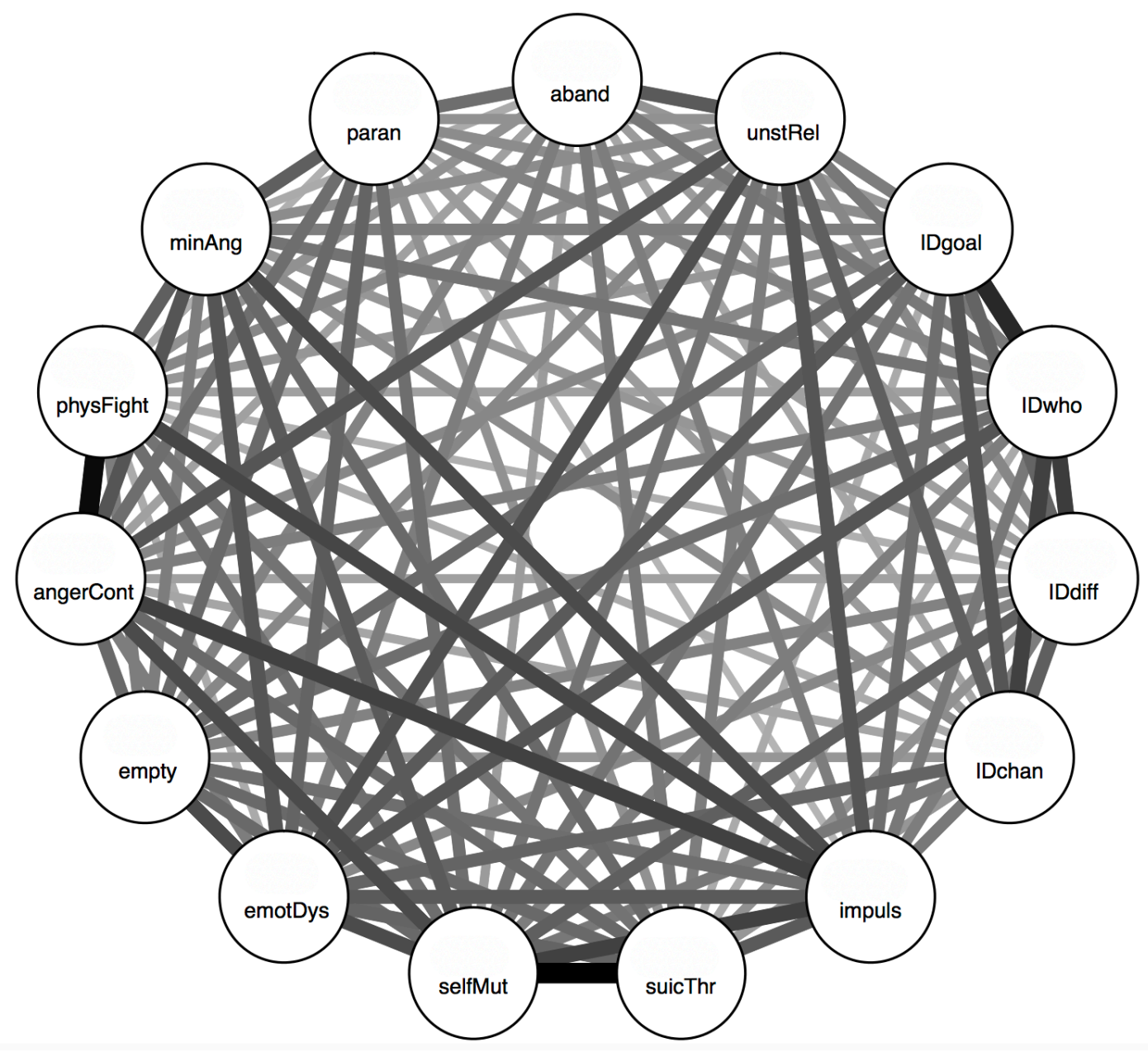

Figure 1. Correlation network of BPD symptoms 


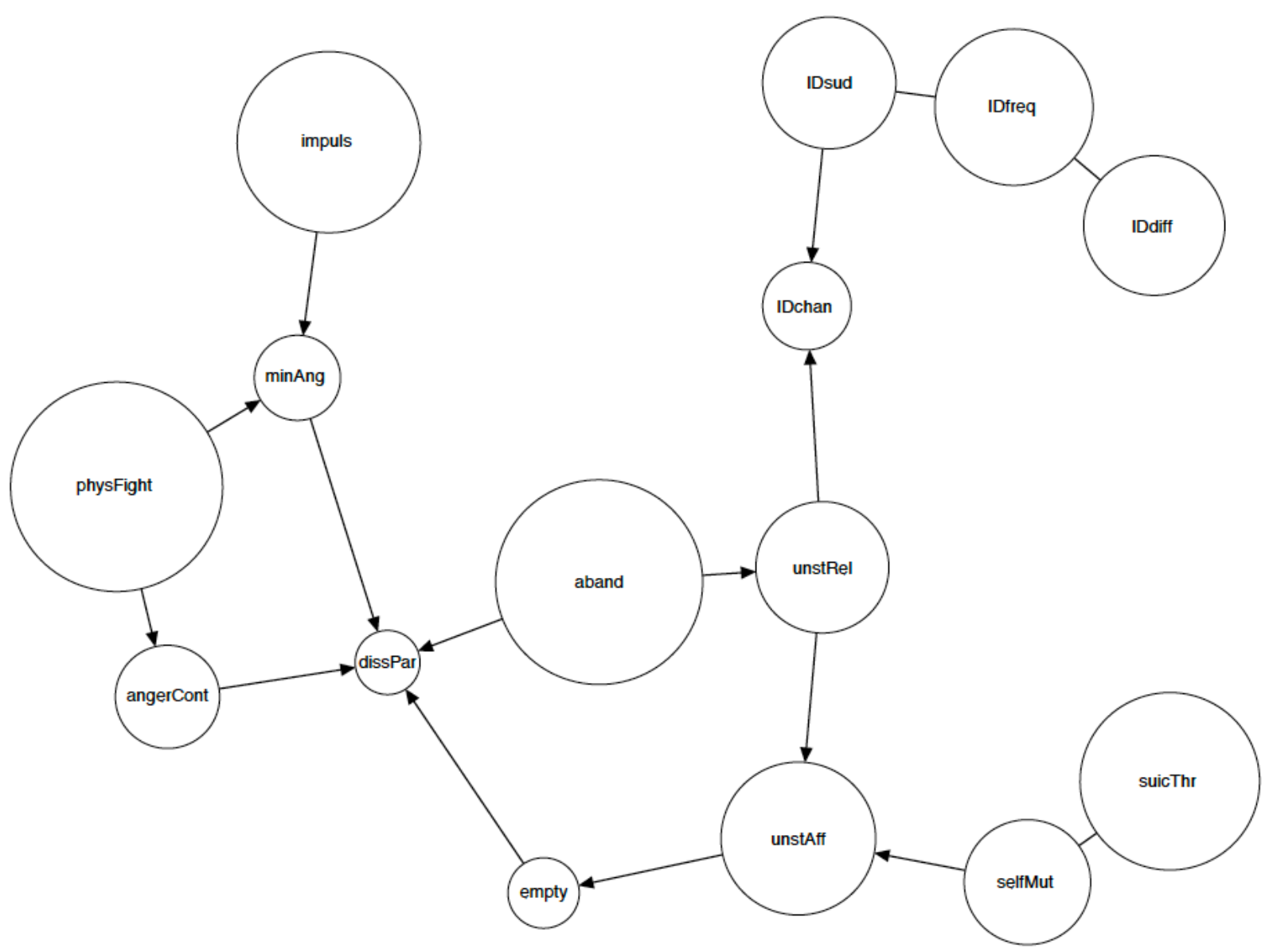

Figure 2. Networks for symptoms of BPD based on the fourth edition of the Diagnostic and Statistical Manual of Mental Disorders (DSM-IV) as a part of the SCID-II for personality disorder. All values are $p<.05$. See Table 1 for definitions of abbreviated terms. 
Table 1. Abbreviations and number of patients meeting criteria per symptom

\begin{tabular}{llll}
\hline \multicolumn{1}{l}{ Question number in } & Abbreviation & & Hierarchy Value \\
\cline { 1 - 2 } 90 & aband & 0.13 \\
91 & unstRel & 0.06 \\
92 & IDSud & 0.06 \\
93 & IDFreq & 0.09 \\
94 & IDdiff & 0.07 \\
95 & IDchan & 0.03 \\
96 & impuls & 0.12 \\
97 & suicThr & 0.10 \\
98 & selfMut & 0.06 \\
99 & UnstAff & 0.08 \\
100 & empty & 0.01 \\
101 & angerCont & 0.04 \\
102 & physFight & 0.13 \\
103 & minAng & 0.02 \\
104 & DissPar & 0.01 \\
\hline
\end{tabular}

Table 2. Hierarchy values of symptoms 
Table 2. Hierarchy values of symptoms

\begin{tabular}{|c|c|c|}
\hline $\begin{array}{l}\text { Question number in } \\
\text { SCID-II }\end{array}$ & Abbreviation & $\underline{\text { Hierarchy Value }}$ \\
\hline 90 & aband & 0.13 \\
\hline 91 & unstRel & 0.06 \\
\hline 92 & IDSud & 0.06 \\
\hline 93 & IDFreq & 0.09 \\
\hline 94 & IDdiff & 0.07 \\
\hline 95 & IDchan & 0.03 \\
\hline 96 & impuls & 0.12 \\
\hline 97 & suicThr & 0.10 \\
\hline 98 & selfMut & 0.06 \\
\hline 99 & UnstAff & 0.08 \\
\hline 100 & empty & 0.01 \\
\hline 101 & angerCont & 0.04 \\
\hline 102 & physFight & 0.13 \\
\hline 103 & minAng & 0.02 \\
\hline 104 & DissPar & 0.01 \\
\hline
\end{tabular}

